\title{
Subwavelength plasmonic waveguide structures based on slots in thin metal films
}

\author{
Georgios Veronis and Shanhui Fan \\ Department of Electrical Engineering, Stanford University, Stanford, California 94305 \\ gveronis@stanford.edu
}

\begin{abstract}
We demonstrate the existence of a bound optical mode supported by a slot in a thin metallic film deposited on a substrate, with slot dimensions much smaller than the wavelength.

(C)2006 Optical Society of America

OCIS codes: $130.2790,240.6680,260.2110$.
\end{abstract}

\section{Introduction}

Waveguide structures which support highly-confined optical modes are important for achieving compact integrated photonic devices [1,2]. In particular, plasmonic waveguides have shown the potential to guide subwavelength optical modes. Several different plasmonic waveguiding structures have been proposed, such as metallic nanowires [3,4] and metallic nanoparticle arrays [5,6]. However, these structures support a highly-confined mode only near the surface plasmon frequency. In this regime, the optical mode typically has low group velocity and short propagation length.

\section{Guided subwavelength plasmonic mode supported by a slot in a thin metal film}

We first demonstrate the existence of a bound optical mode supported by an air slot in a thin metallic film deposited on a substrate, with slot dimensions much smaller than the wavelength. The modal size is almost completely dominated by the near field of the slot. Consequently, the size is very small compared with the wavelength, even when the dispersion relation of the mode approaches the light line of the surrounding media. In addition, the group velocity of this mode is close to the speed of light in the substrate, and its propagation length is tens of microns at the optical communication wavelength. Thus, such a waveguide could be potentially important in providing an interface between conventional optics and subwavelength electronic and optoelectronic devices.

Calculations are performed using the finite-difference frequency-domain (FDFD) method [7]. In Fig. 1(a) we show the dispersion relation of the fundamental mode of the plasmonic slotline. The width $w$ and thickness $t$ of the slot are $50 \mathrm{~nm}$, and the substrate material is silica $\left(n_{\mathrm{s}}=1.44\right)$. We observe that such a structure supports a bound mode that lies below the silica light line in a wide frequency range. In Fig. 1(b) we show the propagation length of the fundamental mode of the plasmonic slotline as a function of wavelength. The propagation length decreases as the wavelength decreases. At the optical communication wavelength of $1.55 \mu \mathrm{m}$ the propagation length is $\sim 20 \mu \mathrm{m}$.
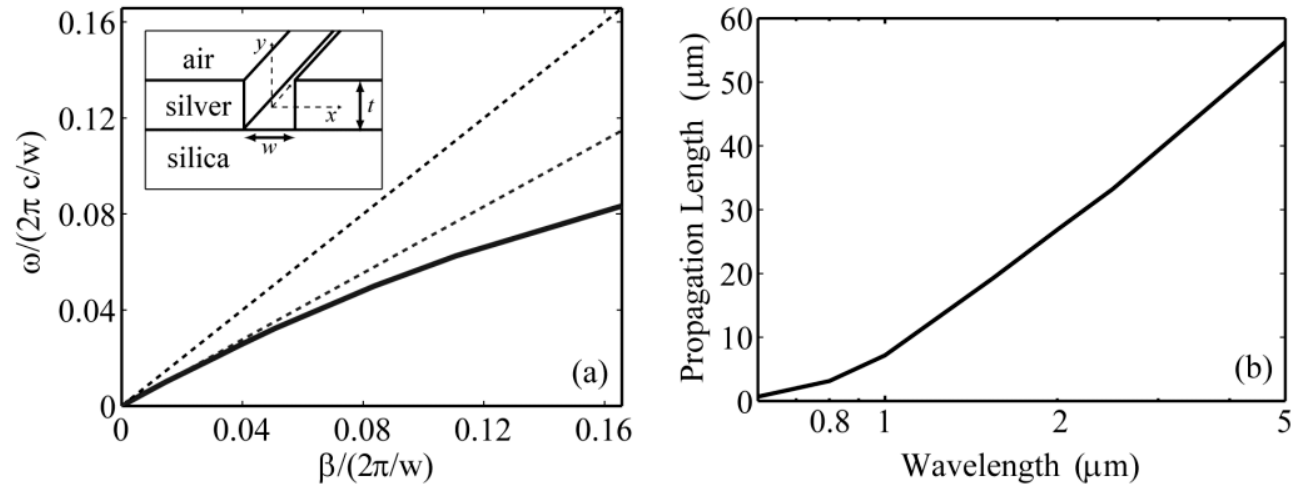

Fig. 1. (a) Dispersion relation of the fundamental mode of the plasmonic slotline (solid curve) for $w, t=50 \mathrm{~nm}$ (see inset). Also shown by thin dotted lines are the light lines of air and silica. (b) Propagation length of the fundamental mode of the plasmonic slotline as a function of wavelength for $w, t=50 \mathrm{~nm}$. 


\section{JThC94.pdf}

\section{Bends and splitters in metal-dielectric-metal subwavelength plasmonic waveguides}

We also investigate the performance of bends and power splitters in plasmonic slot waveguides. We show that, even though the waveguides are lossy, bends and splitters with no additional loss can be designed over a wavelength range that extends from DC to near-infrared, when the bend and splitter dimensions are much smaller than the propagation length of the optical mode. We account for this effect with an effective characteristic impedance model based upon the real dispersion relation of the plasmonic waveguide structures.

In Fig. 2(a), we show the calculated bend transmission coefficient as a function of wavelength. We observe that at long wavelengths there is no bending loss. The operating wavelength range widens as $d$ decreases. In Fig. 2(b), we show the calculated reflection coefficient $R$ of a plasmonic slot $T$-shaped splitter at $1.55 \mu \mathrm{m}$ as a function of $d_{\text {in }} / d_{\text {out, }}$, where $d_{\text {out }}=50 \mathrm{~nm}$ is the thickness of the two output waveguide branches (inset of Fig. 2(b)). In Fig. 2(b), we also show the reflection coefficient $R_{\mathrm{i}}$ calculated based on the characteristic impedance of the waveguides and transmission-line theory. We observe that there is very good agreement between $R_{\mathrm{i}}$ and the exact reflection coefficient $R$ calculated using FDFD. This agreement suggests that the concept of characteristic impedance for plasmonic slot waveguides is indeed valid and useful.
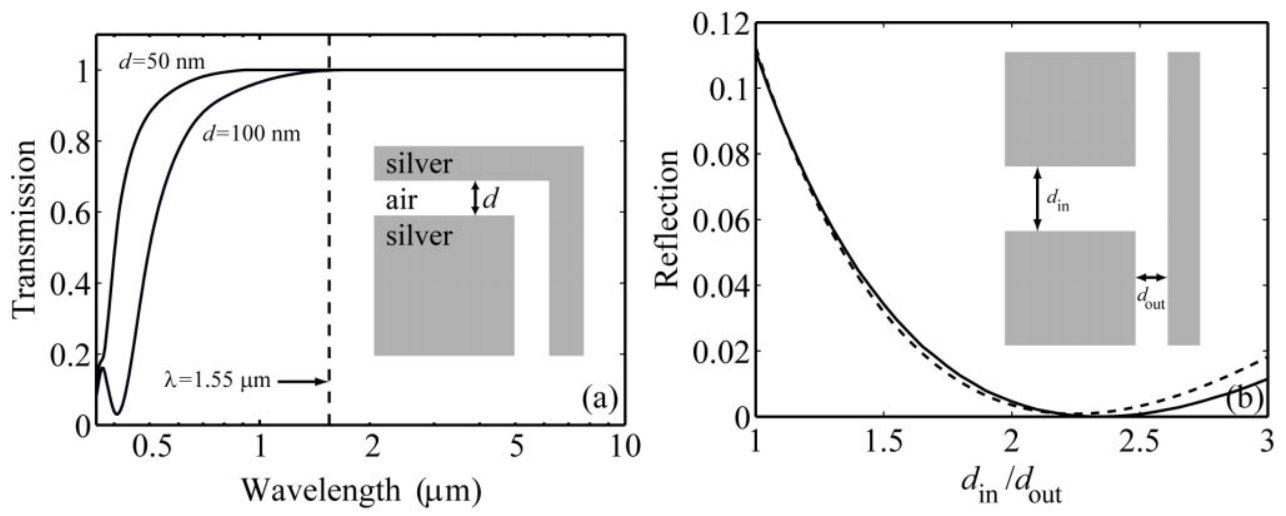

Fig. 2. (a) Transmission spectra of a sharp plasmonic slot waveguide bend (shown in the inset). Results are shown for $d=50,100 \mathrm{~nm}$. The vertical dashed line marks the optical communication wavelength of $1.55 \mu \mathrm{m}$. (b) Reflection coefficient $R$ of a $T$-shaped splitter (shown in the inset) as a function of $d_{\mathrm{in}} / d_{\text {out }}$ at $1.55 \mu \mathrm{m}$. We also show with dashed line the reflection coefficient $R_{\mathrm{i}}$ calculated based on the characteristic impedance of the plasmonic slot waveguides and transmission-line theory. Results are shown for $d_{\text {out }}=50 \mathrm{~nm}$.

\section{References}

[1] J. Takahara, S. Yamagishi, H. Taki, A. Morimoto, and T. Kobayashi, "Guiding of a one-dimensional optical beam with nanometer diameter," Opt. Lett. 22, pp. 475-477, 1997.

[2] V. R. Almeida, Q. F. Xu, C. A. Barrios, and M. Lipson, "Guiding and confining light in void nanostructure," Opt. Lett. 29, pp. 1209-1211, 2004.

[3] J. C. Weeber, A. Dereux, C. Girard, J. R. Krenn, and J. P. Goudonnet, "Plasmon polaritons of metallic nanowires for controlling submicron propagation of light," Phys. Rev. B 60, pp. 9061-9068, 1999.

[4] J. R. Krenn, B. Lamprecht, H. Ditlbacher, G. Schider, M. Salerno, A. Leitner, and F. R. Aussenegg, "Non-diffraction-limited light transport by gold nanowires," Europhys. Lett. 60, pp. 663-669, 2002.

[5] M. L. Brongersma, J. W. Hartman, and H. A. Atwater, "Electromagnetic energy transfer and switching in nanoparticle chain arrays below the diffraction limit," Phys. Rev. B 62, pp. R16356-R16359, 2000.

[6] S. A. Maier, P. G. Kik, H. A. Atwater, S. Meltzer, E. Harel, B. E. Koel, and A. A. G. Requicha, "Local detection of electromagnetic energy transport below the diffraction limit in metal nanoparticle plasmon waveguides," Nat. Mater. 2, pp. 229-232, 2003.

[7]. S. D.Wu and E. N. Glytsis, "Finite-number-of-periods holographic gratings with finite-width incident beams: analysis using the finite-difference frequency-domain method," J. Opt. Soc. Am. A 19, pp. 2018-2029, 2002. 